\title{
Effects of the Degree of Hydration of Water Swollen Poly(vinyl alcohol) Films on Their Iodine Complexation
}

\author{
Yushi OISHI and Keizo MIYASAKA \\ Department of Textile and Polymeric Materials, Tokyo Institute of Technology, \\ Ookayama, Meguro-ku, Tokyo 152, Japan
}

(Received August 2, 1985)

\begin{abstract}
PVA films with a wide span of hydration (swelling) were soaked at different temperatures in iodine aqueous solutions to make the iodine-PVA complex, with a particular concern for the effects of the degree of hydration (D.H.) on the complex formation. The visible absorption maximum $\lambda_{\max }$ increased with D.H., while it decreased with temperature. The equilibrium amount of the complex increased with D.H. at a given concentration of iodine below $20^{\circ} \mathrm{C}$. However, the effects of $\mathrm{D}$. $\mathrm{H}$. on the amount of complex deteriorated with increasing temperature and at $45^{\circ} \mathrm{C}$ became reversed to those at low temperature. When the samples soaked at $5^{\circ} \mathrm{C}$ were heated in the solution, the amount of complex suddenly began to decrease at $20^{\circ} \mathrm{C}$; this corresponded to the sudden stress relaxation of water swollen PVA films. This transition seems to have great effect on the complexation.
\end{abstract}

KEY WORDS Complex Formation / Poly(vinyl alcohol) / Water Swollen Film / Iodine / Iodine-Poly(vinyl alcohol) Complex / Degree of Hydration / Visible Spectrum / Stress Relaxation / Transition /

Poly(vinyl alcohol) (PVA) forms a complex with iodine, changing the color from brown to blue. ${ }^{1,2}$ The most important application of the complex is use as sheet polarizers with a high quality of dichroism. ${ }^{3}$ The polarizer is usually prepared by soaking PVA films in the iodinepotassium iodide solution with boric acid and by subsequent drawing to cause a high degree of uniaxial orientation. The iodine-PVA complexation occurs both in the aqueous solution and swollen gel states of PVA depending on the iodine concentration and temperature. For example, in solution, the amount of complex increases with increasing concentrations of PVA, iodine and boric acid. ${ }^{4}$ The complex disappears during heating and again appears during cooling, resulting in color changes between blue and brown. ${ }^{2,5,6}$ The extension of PVA gels enhances the complexation, ${ }^{5,7}$ suggesting that the extended conformation of
PVA chain segments is favorable for complexation. The strain-induced complexation is reversed on removal of the strain. ${ }^{7}$ Many studies have been made also on the structure of the complex. For example, Zwick hypothesized a structure in which a linear polyiodine chain is included by helical PVA chains going around, ${ }^{4}$ according to the structure of the iodine-amylose complex. ${ }^{8}$ Inagaki et al. ${ }^{9}$ support this structure with the similarity between the raman spectra of iodine-PVA and iodine-amylose complexes. On the other hand, Rundle et al. ${ }^{10}$ proposed another one in which a polyiodine chain lies parallel to an extended part (segment) of a PVA chain. Tebelev ${ }^{11}$ supports Rundle's model and further proposes that the iodine chain is surrounded by several extended PVA segments. In spite of these propositions, the structure has not been explicitly determined. We have been interested in 
the structure and properties of water swollen PVA films with emphasis on their relation to the degree of hydration D.H. ${ }^{7}$ The effects of D.H. on the complexation are our concern in this study. In solution, the increase in the PVA concentration enhances the complexation. ${ }^{4}$ If this is still satisfied in the swollen film, the smaller D.H. is favorable for the complexation. Further, the extension of network chains caused by swelling may help the segments to participate in the complex formation. On the other hand, PVA chains in the gel must be under a large constraint resulting mainly from the crystal interfaces, which may have some inhibitive effect on the complexation. These considerations made us expect the complicated features of the effects of D.H. on the complex formation.

In the present paper, the effects of D.H. on the complexation in swollen films are studied at different temperatures and concentrations of iodine in the soaking solution.

\section{EXPERIMENTAL}

PVA (Koso Chem. Co., DP $=2000$, degree of hydrolysis $=0.99$ ) was used after further saponification with sodium methoxide in $\mathrm{MeOH}$. The IR spectrum of the PVA detected no trace of acetate groups. Films $50 \mu \mathrm{m}$ thick were cast from a $7 \mathrm{wt} \%$ aqueous solution of the PVA on PMMA plates in air at $20^{\circ} \mathrm{C}$, followed by annealing to get different D.H.s of the specimens. The annealing temperature ranged from $100^{\circ} \mathrm{C}$ to $180^{\circ} \mathrm{C}$ in vacuo and the time was 10 minutes. The D.H. defined as the volume fraction of water in a wet PVA film, was estimated at each soaking temperature using the following equation,

$$
\text { D.H. }=\left(M_{\mathrm{s}}-M_{\mathrm{d}}\right) /\left(\left(M_{\mathrm{s}}-M_{\mathrm{d}}\right)+M_{\mathrm{d}} / 1.3\right)
$$

where $M_{\mathrm{s}}$ and $M_{\mathrm{d}}$ are the weights of a film at the equilibrium swollen and dry states, respectively, and 1.3 is the specific gravity of PVA. Iodine and potassium iodide (Yanagishima Pharm. Co., GR grade) were used with- out further purification. Iodine-potassium iodide aqueous solutions with a mol ratio of $1: 2$ were prepared for the soaking of PVA films. The iodine concentration in the solution ranged from $3 \times 10^{-4}$ to $4.5 \times 10^{-3} \mathrm{moll}^{-1}$ which was much less than that in the actual polarizer production. For certification of complexation and evaluation of the amount of complex, the visible ray spectra of the soaked specimens were measured in the wet state in a range from $400 \mathrm{~nm}$ to $700 \mathrm{~nm}$ by a HitachiPerkin-Elmer 139 spectrometer. It took 3 minutes for each measurement of the spectrum, during which the temperature was controlled by making water at a given temperature run through the bottom of the sample cell. In this paper, the spectrum of PVA gels soaked for 10 minutes in the solution of the highest iodine concentration that did not cause any noticeable complexation in about $20 \mathrm{~min}$ utes at a given temperature was assumed to give the shape of the base line for all the spectra with the complex obtained at the given temperature. First, the spectrum of a specimen without the complex thus soaked for $10 \mathrm{~min}$ utes at a given temperature was fitted at $400 \mathrm{~nm}$ to a given spectrum with complexation obtained at the same temperature, assuming that the complex had no absorbances at $400 \mathrm{~nm}$. Using the base line, we regarded the absorbances at $\lambda_{\max }$ as the quantity describing the amount of complex. Since the relationships between the absorbance and the amount of complex has not yet been obtained, the absorbance normalized by the thickness of wet sample was used in place of the amount of complex in this paper. Thus, the absorbance gives the relative amount of complex normalized by a unit volume of wet sample.

\section{RESULTS AND DISCUSSION}

Figure 1 shows the effects of iodine concentration in the soaking solution on the absorption spectra of a PVA gel film of 
D.H. $=0.51$ soaked at $5^{\circ} \mathrm{C}$ for $24 \mathrm{~h}$ which was long enough for equilibrium to be reached. A broad absorption with a maximum at about $600 \mathrm{~nm}$ already assigned to the iodine-PVA complex ${ }^{12}$ intensified with increasing concentration. The increase of the concentration seemed also to increase the wavelength $\lambda_{\max }$ at the absorption maximum, although it was difficult to estimate the exact value because of the broadness of the spectrum. The same changes as shown in Figure 1 were also observed for different D.H.s of the specimens. The $\lambda_{\max }$ depends on the structure of the complex, while the absorbance at the $\lambda_{\max }$ does on the amount of complex.

Figure 2 shows the relationships between $\lambda_{\max }$ and D.H. obtained at different iodine concentrations in the soaking solution at $30^{\circ} \mathrm{C}$. The $\lambda_{\max }$ markedly shifts to the longer side with increasing D.H. and iodine concentration; the effect of D.H. is particularly large.

Figure 3 shows the relationships between $\lambda_{\max }$ and D.H. at various temperatures, for given iodine concentrations of $1 \times 10^{-3} \mathrm{moll}^{-1}$ at $5^{\circ} \mathrm{C}, 2 \times 10^{-3} \mathrm{moll}^{-1}$ at $15^{\circ} \mathrm{C}, 3 \times 10^{-3}$ moll $1^{-1}$ at $25^{\circ} \mathrm{C}$, and $4 \times 10^{-3} \mathrm{moll}^{-1}$ at 30 and $45^{\circ} \mathrm{C}$. The $\lambda_{\max }$ increases with D.H. at all temperatures, and the temperature dependence of $\lambda_{\max }$ is more remarkable at high D.H. It is noted, however, that the D.H. dependence of $\lambda_{\max }$ is very small at $45^{\circ} \mathrm{C}$. These relations hold even at different iodine concentrations since $\lambda_{\max }$ shifts to the longer side with increasing iodine concentration as shown in Figure 2.

The $\lambda_{\max }$ of the iodine-PVA complex in the absence of boric acid has not yet been shown to be explicitly related to the length of poly iodine (numbers of iodine atoms in a complex). It is known that the presence of boric acid markedly increases the $\lambda_{\max }$ of the complex. ${ }^{4}$ The spectra of our iodine-PVA gels were very similar to those of the iodine-poly(vinyl acetate) (PVAc) complexes reported by Hayashi et al. ${ }^{13}$ On the basis of Raman data, they concluded that the $\lambda_{\max }$ due to $\mathrm{I}_{5}^{-}$iodine

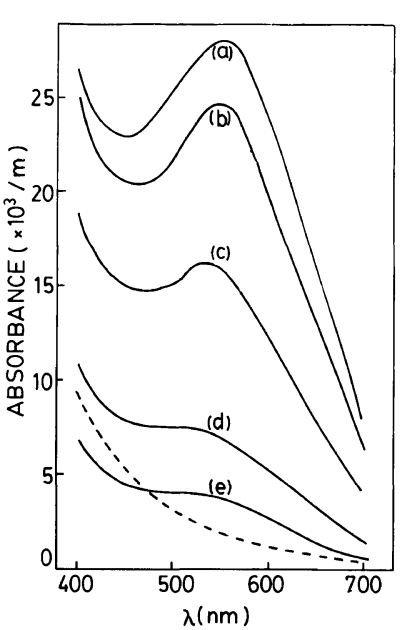

Figure 1. Absorption spectra of iodine sorbed PVA films at various iodine concentrations: (a) $3.0 \times 10^{-3}$ moll-1; (b) $2.5 \times 10^{-3} \mathrm{moll}^{-1}$; (c) $2.0 \times 10^{-3} \mathrm{moll}^{-1}$; (d) $1.5 \times 10^{-3} \mathrm{moll}^{-1}$; (e) $1.0 \times 10^{-3} \mathrm{moll}^{-1}$ at $5^{\circ} \mathrm{C}$, together with the base line $\left(2.0 \times 10^{-3} \mathrm{moll}^{-1}, 10 \mathrm{~min}\right)$ given by a dashed line.

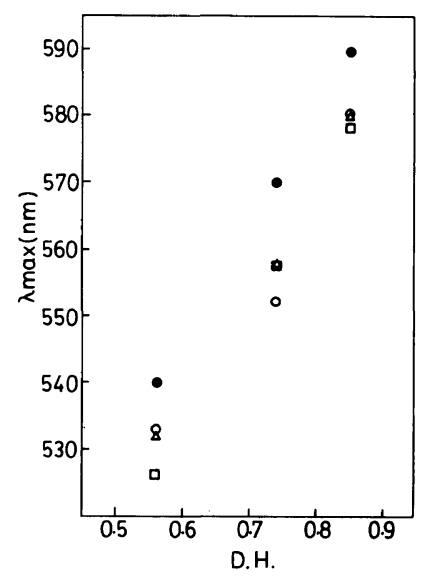

Figure 2. The absorption maximum $\lambda_{\max }$ as a function of D.H. at various iodine concentrations: (O)

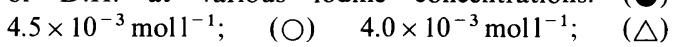
$3.5 \times 10^{-3} \mathrm{moll}^{-1}$; (口) $3.0 \times 10^{-3} \mathrm{moll}^{-1}$ at $30^{\circ} \mathrm{C}$.

complex is $620 \mathrm{~nm}$ for the blue-colored iodinePVAc complex, $670 \mathrm{~nm}$ for iodine-PVA complex in the presence of boric acid and $600 \mathrm{~nm}$ for the iodine-amylose complex. On the other hand, the $\lambda_{\max }$ of $510 \mathrm{~nm}$ for red-violet colored iodide-PVAc complex has been assigned to $\mathrm{I}_{3}^{-}$ from the analogy of the Raman spectrum to 


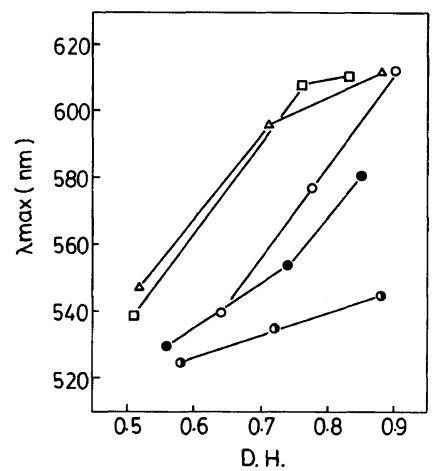

Figure 3. The absorption maximum $\lambda_{\max }$ as a function of D.H. at various temperatures: $(\square) 5^{\circ} \mathrm{C} ;(\triangle) 15^{\circ} \mathrm{C}$; (○) $25^{\circ} \mathrm{C}$; (○) $30^{\circ} \mathrm{C}$; (○) $45^{\circ} \mathrm{C}$.

that of polycrystalline (benzamide) ${ }_{2} \mathrm{H}^{+} \mathrm{I}_{3}^{-}$used as the model substance. These considerations lead us to suppose that the shift of $\lambda_{\max }$ from $520 \mathrm{~nm}$ to $620 \mathrm{~nm}$, caused by increased D.H. and decreased temperature, may be attributed to the increase in the number of iodine atoms in a polyiodine chain. It should be remarked, however, that the shift occurs gradually and continuously with change in D.H. and temperature, implying that the fraction of the $\mathrm{I}_{5}^{-}$complex gradually increases but not transition-like.

Figure 4 shows the absorbance at $\lambda_{\max }$ which is proportional to the equilibrium amount of complex, as functions of iodine concentration and D.H. at $5,45^{\circ}$ C. According to Figure 4, the amount of complex increases with increasing iodine concentration at both temperatures. However, the ratio of the amount of complex to the iodine concentration is much dependent on D.H. and temperature. It is interesting to note that the D.H. dependence of the ratio is not monotonous; at $5^{\circ} \mathrm{C}$, the larger the D.H, the larger the ratio, and at $45^{\circ} \mathrm{C}$, the effect of D.H. on the ratio takes the reverse order of that at low temperature. The curves shown in Figure 4 and curves obtained at 15, 25, and $30^{\circ} \mathrm{C}$ were found to be expressed by the following equation.

$$
Q_{\mathrm{c}}=(K C)^{A}
$$

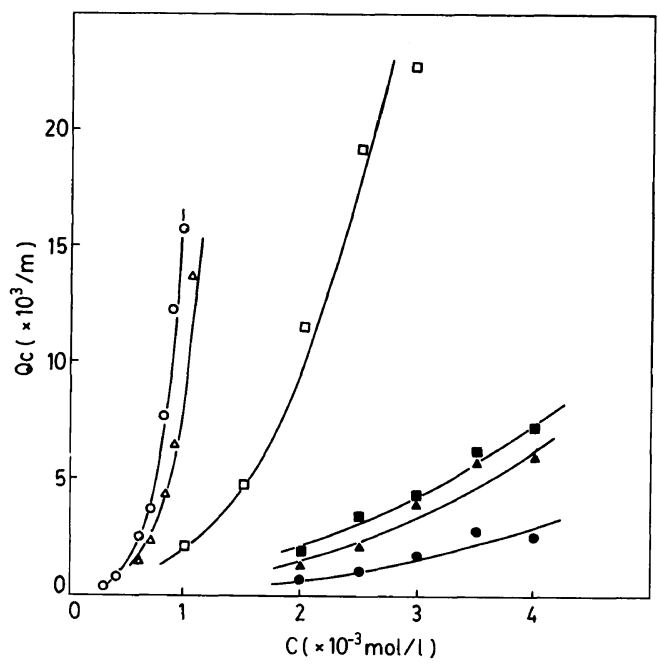

Figure 4. The relation between the amount of complex $Q_{\text {c }}$ and the iodine concentration $C$ in the solution as a function of D.H.: $(\bigcirc) 0.83 ;(\triangle) 0.76$; $(\square) 0.51$ at $5^{\circ} \mathrm{C}$, (O) $0.88 ;(\Delta) 0.72 ;(\square) 0.58$ at $45^{\circ} \mathrm{C}$.

where $Q_{\mathrm{c}}$ is the absorbance at $\lambda_{\max }$ which is proportional to the amount of complex formed and $C$ is the iodine concentration of soaking solution. $K$ was found to be a constant of $1.2 \times 10^{4}$.

Figure 5 shows the eq 2 plot of data at $5^{\circ} \mathrm{C}$, indicating that the relation is well satisfied. Equation 2 means that the larger the parameter $A$, the larger is the amount of complex is at a given iodine concentration.

In Figure 6, the parameter $A$ is plotted against D.H, indicating that $A$ has a linear relationship with D.H. At any D.H., decreasing temperature always increased the amount of the complex. This means that less thermal agitation of chains is favorable for complex formation. It should be remarked that the $A$ is for the equilibrium amount of complex, and therefore the temperature dependence of $A$ is free from the temperature effect on the rate of complex formation. In Figure 6, the parameter $A$ changes almost linearly with increasing D.H. at all temperatures, although the slope decreases with increasing temperature and becomes even negative at $45^{\circ} \mathrm{C}$.

In the case of homogeneous networks such 


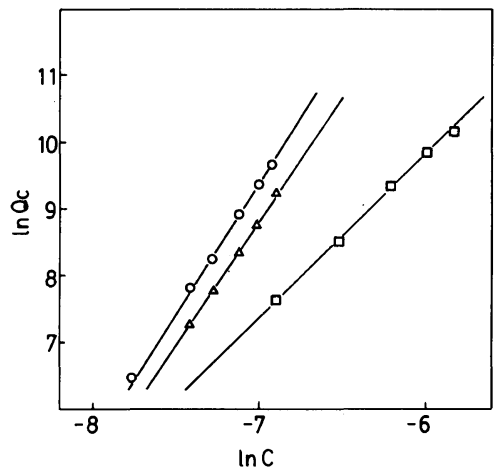

Figure 5. The relation between the $\log$ of the amount of complex $Q_{\mathrm{c}}$ and the $\log$ of the iodine concentration $C$ at $5^{\circ}$ C. D.H.: (O) 0.83 ; $\triangle$ ) 0.76 ; $\square$ ) 0.51 .

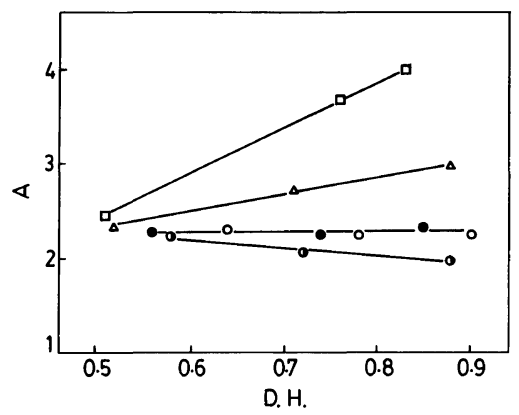

Figure 6. Parameter $A$ as a function of D.H. at various temperatures: $(\square) 5^{\circ} \mathrm{C}$; $\triangle$ ) $15^{\circ} \mathrm{C}$; (○) $25^{\circ} \mathrm{C}$; (○) $30^{\circ} \mathrm{C}$; (ब) $45^{\circ} \mathrm{C}$.

as a cross-linked rubber, the equilibrium degree of swelling $q_{\mathrm{m}}$ depends on the molecular weight of network chains $M_{\mathrm{c}}$. According to Flory, ${ }^{14}$ the following relation is expected for a given rubber network-solvent system,

$$
M_{\mathrm{c}} \propto q_{\mathrm{m}}^{5 / 3}
$$

This quantitative relation is not expected in our heterogeneous networks in which crystallites play the role of junction points. We may, however, assume that the averaged molecular weight of network chains may be larger in larger D.H. gels than in smaller D.H. ones. This leads us to suppose that the parameter $A$ may depend on the mobility of PVA chains in the gel determined by their thermal agitation and the length of network chains. The results showed that the longer network chain is favor- able for complex formation, unless thermal agitation is too intense. However, it should be remarked that the fact that the parameter $A$ decreases with increasing D.H. at $45^{\circ} \mathrm{C}$ should be discussed together with the following experimental results. The following experiments were carried out in order to study the nature of the complex after being soaked in a solution of $2.5 \times 10^{-3}$ moll $^{-1}$ iodine concentration at $5^{\circ} \mathrm{C}$ until the equilibrium amount of complex was formed, specimens in the same solution were heated stepwise and kept at each temperature for 30 minutes, and the amount of complex was followed by a photometer.

Figure 7 shows the absorbance of the complex as a function of temperature, indicating that the amount of complex remaining almost constant below $20^{\circ} \mathrm{C}$ begins to decrease linearly with increasing temperature above $20^{\circ} \mathrm{C}$. Finally the complex disappears completely at $75^{\circ} \mathrm{C}$ and $60^{\circ} \mathrm{C}$ in the specimens of D.H. $=0.51$ and 0.83 , respectively. At a given temperature above $20^{\circ} \mathrm{C}$, the amount of complex in the less D.H. specimens is larger than in the larger D.H. ones. The $\lambda_{\max }$ of these specimens remains almost unchanged during heating, as is also shown in Figure 7, which is contrast to the results shown in Figure 3 that $\lambda_{\max }$ decreases with increasing soaking temperature at which the complex is formed. The decrease of the amount of complex with increasing temperature in Figure 7 is qualitatively in accordance with the temperature dependence of the parameter $A$ as seen in Figure 6. However, the decrease of the parameter $A$ occurred even below $20^{\circ} \mathrm{C}$, while in Figure 7, the decrease of the amount of complex begins at $20^{\circ} \mathrm{C}$. That the temperature dependence of the amount of complex in Figure 7 does not completely accord with that of the parameter $A$ indicates a hysteresis phenomenon due to the stability of the complex depending on the soaking temperature.

Figure 8 shows the stress of swollen PVA films under a $20 \%$ extension as a function of temperature. The heating conditions in Figure 


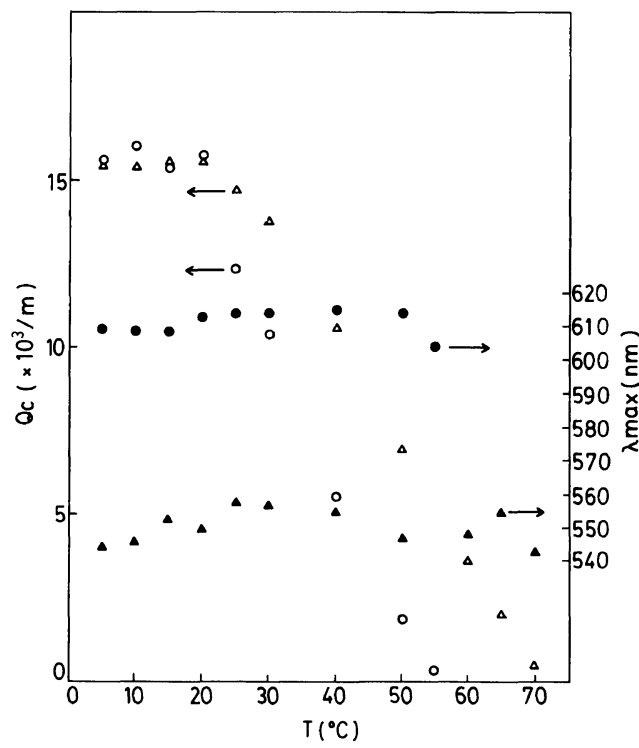

Figure 7. Decomposion behaviour of iodine-PVA complex for different D.H.s: $(O, 0) 0.83,1 \times$ $10^{-3} \mathrm{moll}^{-1} ;(\triangle, \Delta) 0.51,2.5 \times 10^{-3} \mathrm{moll}^{-1}$ as a function of temperature.

8 were the same as in Figure 7. In spite of the big differences between the stress values of these samples with different D.H.s, their shapes of stress relaxation curves were very similar to each other. The comparison of Figure 8 with Figure 7 indicates that (1) the stress relaxation becomes remarkable at temperatures close to those where the amount of complex begins to decrease in Figure 7 and (2) the stress relaxation occurs faster in the larger D.H. sample in which the amount of complex decreases faster in Figure 7. These similarities suggest some correlation between them, even if it is difficult to find a quantitative relationship between them. The change of stress with increasing temperature is attributed to the enhanced stress relaxation and the increased entropy force. The sudden beginning of stress relaxation in Figure 8 indicates a discontinuous increase in the mobility of the network chains at the temperature, implying a kind of transition in PVA gels. It has been reported ${ }^{15}$ that the iodine-PVAc complex suddenly disappears when the temperature is over the glass

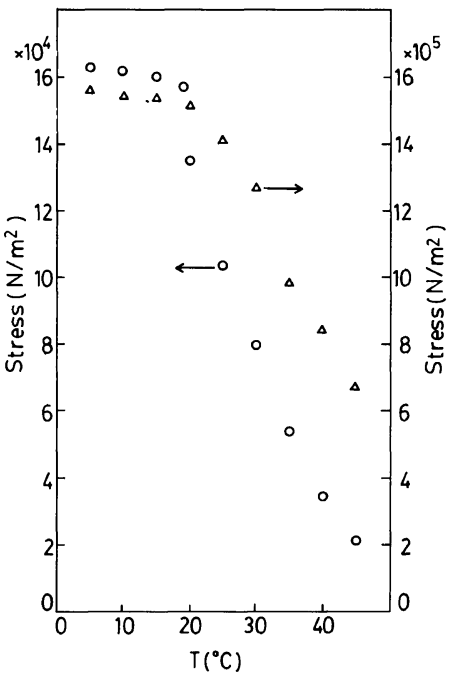

Figure 8. The stress relaxation at a constant length of PVA films for different D.H.s: $(\bigcirc) 0.83 ;(\triangle) 0.51$ as a function of temperature.

transition point of the polymer. In our case, the decrease of the amount of complex occurs gradually, suggesting that the transition at $20^{\circ} \mathrm{C}$ is not the $T_{\mathrm{g}}$ where the chain mobility changes from that of solid to that of liquid. Figure 8 shows the decrease of the amount of complex in Figure 7 to be induced by the increase in the chain mobility due to thermal agitation, and that the thermal agitation is more intense in the higher D.H. PVA gel than in the lower D.H. gel. If the decrease in the amount of complex in Figure 7 is due to decomposition of complex, the decomposition point of the stablest complex formed in the specimens of D.H. $=0.51$ is about $75^{\circ} \mathrm{C}$ and that in the D.H. $=0.83$ specimens is $60^{\circ} \mathrm{C}$, while that of the most unstable complex of these specimens is about $20^{\circ} \mathrm{C}$. This span of the decomposition point of the complex may be too wide even if the heterogeneous structures of the gel and the complex are taken into considerations. However, if structural changes to enhance the mobility of the PVA segment such as the melting of very small crystallites gradually occur in the gel above $20^{\circ} \mathrm{C}$, this widely distributed decomposition of the com- 
plex is possible. The results indicates that the effects of the transition of PVA film must be taken into consideration when the effects of D.H. on the complexation are studied. It is necessary to investigate what occurs above $20^{\circ} \mathrm{C}$ in water swollen PVA gels, in order to discuss further this problem. Thus, we are now preparing a small angle X-ray scattering experiment to study the nature of the transition at $20^{\circ} \mathrm{C}$ of PVA gels.

\section{CONCLUSIONS}

The formation, decomposition and structure of iodine-PVA complex largely depend on the network structure of PVA film described by D.H. and on the thermal motion of network chains. Both the length (number of iodine atoms in a complex) and the number of complex become larger when the network chain is longer and the thermal mobility is lower. The mobility of molecular chains enhanced by melting of crystallites during heating decreases the amount of the complex.

\section{REFERENCES}

1. W. O. Herrmann and W. Haehnel, Ber. Deutsch. Chem. Ges., 60, 1658 (1927).
2. H. Staudinger, K. Frey, and W. Starch, Ber. Deutsch, Chem. Ges., 60, 1782 (1927).

3. E. H. Land, J. Opt. Soc. Am., 41, 957 (1951); C. D. West and R. Clark Jones, ibid., 41, 982 (1951).

4. M. M. Zwick, J. Appl. Polym. Sci., 9, 2393 (1965); J. Polym. Sci., A-1, 4, 1642 (1966).

5. Y. Tanizaki, T. Kobayashi, and N. Ando, Nippon Kagaku Zasshi, 80, 445 (1959).

6. T. Yokota and Y. Kimura, Makromol. Chem., 185, 749 (1984).

7. Y. Kojima, K. Furuhata, and K. Miyasaka, J. Appl. Polym. Sci., 30, 1617 (1985).

8. K. Freudenberg, E. Schaaf, G. Dumpert, and T. Ploetz, Naturwissenschaften, 27, 850 (1939).

9. F. Inagaki, I. Harada, T. Shimanouchi, and M. Tasumi, Bull. Chem. Soc. Jpn., 45, 3384 (1972).

10. R. E. Rundle, J. F. Foster, and R. R. Baldwin, J. Am. Chem. Soc., 66, 2116 (1944).

11. L. G. Tebelev, G. F. Mikul'skii, Y. E. P. Korchagina; and S. A. Glikman, Vysokomol. Soedin., Ser. A., 7, 1231 (1965).

12. K. Imai and M. Matsumoto, J. Polym. Sci., 55, 335 (1961).

13. S. Hayashi, T. Hirai, N. Hojo, H. Sugeta, and Y. Kyogoku, J. Polym. Sci., Polym. Lett. Ed., 20, 69 (1982).

14. P. J. Flory, "Principles of Polymer Chemistry," Cornell Univ. Press, Ithaca, New York, 1953, Chapter 13.

15. M. Tanaka and K. Mizutani, Angew. Makromol. Chem., 78, 211 (1979); S. Hayashi, C. Kawamura, and M. Takayama, Bull. Chem. Soc. Jpn., 43, 537 (1970). 